\title{
Solution of Harmonic Oscillator Perturbed by Electric Field
}

\author{
Habtamu Dagnaw \\ Department of Physics, Injibara University, PO box 040, Injibara, Ethiopia
}

\begin{abstract}
The aim of this work is to analyze the energy eigen value of harmonic oscillator perturbed by electric field. In order to carry out the analysis, we have used two independent methods, suchas the perturbation method and time independent schrodinger equation. We have calculated the first order energy correction, second order energy correction, and the energy eigen value for time independent schrodinger equation. It has been found that the energy eigen value of harmonic oscillator is the same in two independent methods.
\end{abstract}

Keywords: Harmonic ocillator, Electric Field, Perturbation Method, Eigen value.

DOI: $10.7176 /$ APTA/78-01

Publication date:June $30^{\text {th }} 2019$

\section{Introduction}

The harmonic oscillator is an important model in the study of many problems in the area of physics. However, in contrast to the harmonic oscillator, it cannot be solved analytically, and thus one has to resort to approximation methods for its solution[1-3]. Some Authors have analyzed the energy eigen values of harmonic oscillators using Perturbation method[1]. Safwan A. etal have studied time-independent Schrodinger equation for a charged particle, in the presence of electric potential and vector potential, using He's Homotopy Perturbation Method (HPM). HPM is one of the newest analytical methods to solve linear and nonlinear differential equations. The results show the evidence of simplicity, usefulness, and effectiveness of the HPM for obtaining approximate analytical solutions for the time-independent Schrodinger equation for a charged particle in parallel electric and magnetic fields[4]. Adelaku A. and Abajingin D, have studied the energy eigen values for quantum anharmonics oscillator with quartic perturbation potential using two independent methods, the dirac operator technique and the numerov approach in solving schrodinger equation. The results showed that the two independent approaches tobe closely compatible at high excitation sates, the choice of zeta axis was made to satisfy the boundary condition[5].

The aim of this work is to calculate the energy eigen values of the harmonic oscillator perturbed with electric field, uing two independent methods. We have calculated the first order energy correction, second order energy correction and the exact energy eigen value of harmonic oscillator using time independent schrodinger equation.

\section{Peturbation Method}

In this section, we seek to determine the correction of first order energy and second order energy by using first order and second order energy shift formulas.

\subsection{First order energy shift}

Inorder to find first order energy correction, using the first order energy shift is

$$
E_{n}{ }^{1}=\left\langle\psi_{n}{ }^{0}\left|H^{\prime}\right| \psi_{n}{ }^{0}\right\rangle \text {, }
$$

where

$$
\begin{aligned}
& H^{\prime} \text { is perturbed Hamiltonian of the system } \\
& H^{\prime}=-q E x \text {. }
\end{aligned}
$$

Inserting $\mathrm{Eq}(2)$ into $\mathrm{Eq}(1)$, we get

$$
E_{n}{ }^{1}=-q E\left\langle\psi_{n}{ }^{0}|x| \psi_{n}{ }^{0}\right\rangle
$$

in which

$\mathrm{n}$ is the $\mathrm{n}^{\text {th }}$ eigen function. Employing the ladder operators, we see that

$$
\text { 缷 }=\sqrt{\frac{\hbar}{2 m \omega}}\left[a^{+}+a\right]
$$

Substituting $\mathrm{Eq}(4)$ into $\mathrm{Eq}(3)$, we have

$$
E_{n}{ }^{1}=-q E \sqrt{\frac{\hbar}{2 m \omega}}(\langle n| \text { 湮 }+a|n\rangle) \text {. }
$$

Since

$$
\hat{a}^{+}|n\rangle=\sqrt{n+1}|n+1\rangle
$$


And

$$
\hat{a}|n\rangle=\sqrt{n}|n-1\rangle
$$

Onaccount of $\mathrm{Eq}(6)$ and $\mathrm{Eq}(7), \mathrm{Eq}(5)$ can be put inthis form

$$
E_{n}{ }^{1}=-q E \sqrt{\frac{\hbar}{2 m \omega}}(\sqrt{n+1}\langle n \mid n+1\rangle+\sqrt{n}\langle n \mid n-1\rangle) \text {. }
$$

The properties of kronical delta function

$$
\langle m \mid n\rangle=\delta_{m n}=\left\{\begin{array}{l}
1 \text { ifm }=n \\
0 \text { ifm } \neq n
\end{array}\right.
$$

Onaccounts of $\mathrm{Eq}(6), \mathrm{Eq}(8)$ can be written as

$$
E_{n}^{1}=0
$$

This equation repersentes first order energy correction to the energy eigen value $E_{n}$. The energy eigen value in the first approximation is

$$
\mathrm{E}_{\mathrm{n}}=\mathrm{E}_{\mathrm{n}}{ }^{0}
$$

\subsection{Second order energy shift}

In this section, we seek to obtain the second order energy correction, using the fundamental equation of the second order perturbation theory which is

$$
E_{n}^{2}=\sum_{n \neq m} \frac{\left|\left\langle\psi_{m}{ }^{0}\left|H^{\prime}\right| \psi_{n}{ }^{0}\right\rangle\right|^{2}}{E_{n}{ }^{0}-E_{m}{ }^{0}} \text {. }
$$

Where

$$
\left\langle\psi_{m}{ }^{0}\left|H^{\prime}\right| \psi_{n}{ }^{0}\right\rangle=-q E\langle m|x| n\rangle \text {. }
$$

Onaccounts of $\operatorname{Eq}(4)$, we easily get

$$
\left\langle\psi_{m}{ }^{0}\left|H^{\prime}\right| \psi_{n}{ }^{0}\right\rangle=-q E \sqrt{\frac{\hbar}{2 m \omega}}(\sqrt{n+1}\langle m \mid n+1\rangle+\sqrt{n}\langle m \mid n-1\rangle),
$$

With the delta function, it is important to note that

$\langle m \mid n+1\rangle=\delta_{m, n+1}$

And

$\langle m \mid n-1\rangle=\delta_{m, n-1}$.

Substituting this relation into $\mathrm{Eq}(14)$, can be rewrite this way

$$
\left\langle\psi_{m}{ }^{0}\left|H^{\prime}\right| \psi_{n}{ }^{0}\right\rangle=-q E \sqrt{\frac{\hbar}{2 m \omega}}\left(\sqrt{n+1} \delta_{m, n+1}+\sqrt{n} \delta_{m, n-1}\right),
$$

Substituting Eq (15)into Eq(12), it becomes

$$
E_{n}^{2}=\sum_{n \neq m} \frac{\left|-q E \sqrt{\frac{\hbar}{2 m \omega}}\left(\sqrt{n+1} \delta_{m, n+1}+\sqrt{n} \delta_{m, n-1}\right)\right|^{2}}{E_{n}{ }^{0}-E_{m}{ }^{0}},
$$

But the eigen value of energy of harmonic oscillator is

$$
E_{n}{ }^{0}=\left(n+\frac{1}{2}\right) \hbar \omega \quad \text { And } \quad E_{m}{ }^{0}=\left(m+\frac{1}{2}\right) \hbar \omega
$$

Inserting $\mathrm{Eq}(17)$ into $\mathrm{Eq}(16)$, we have 
$E_{n}^{2}=\frac{(q E)^{2}}{2 m \omega^{2}} \sum_{n \neq m} \frac{\left|\left(\sqrt{n+1} \delta_{m, n+1}+\sqrt{n} \delta_{m, n-1}\right)\right|^{2}}{n-m}$,

Employing delta function identity, finally we get

$$
E_{n}{ }^{2}=\frac{-(q E)^{2}}{2 m \omega^{2}} \text {. }
$$

$\mathrm{Eq}(19)$ represents second order energy correction. The energy eigen value in the second approximation is

$$
E_{n}=\left(n+\frac{1}{2}\right) \hbar \omega-\frac{(q E)^{2}}{2 m \omega^{2}}
$$

Where $\mathrm{n}$ is a positive integer $(\mathrm{n}=0,1,2,3, \ldots \ldots)$. The second order perturbation theory gives the exact correction to the energy.

\section{Time independent schrodinger Equation}

We now to obtain the exact energies of harmonic oscillators perturbed by electric field using time independent schrodinger equation. Consider a one dimensional harmonic oscillator in a constant electric field. The time independent schrodinger equation is defiend by

$\left(H^{0}+H^{\prime}\right) \psi=E \psi$,

Where $H^{0}$ is unperturbed Hamiltonian for harmonic oscillator

$$
H^{0}=\frac{-\hbar^{2}}{2 m} \frac{d^{2}}{d x^{2}}+\frac{1}{2} m \omega x^{2}
$$

And

$$
H^{\prime}=-q E x
$$

Substituting $\mathrm{Eq}(22), \mathrm{Eq}(23)$ into $\mathrm{Eq}(21)$, we have

$$
\frac{-\hbar^{2}}{2 m} \frac{d^{2} \psi}{d x^{2}}+\left(\frac{1}{2} m \omega x^{2}-q E x\right) \psi=E \psi
$$

We introduce the new variable

$$
x^{\prime}=x-\frac{q E}{m \omega^{2}} \Rightarrow x=x^{\prime}+\frac{q E}{m \omega^{2}}
$$

Considering first the potential part of the schrodinger equation and changing the variables, we have

$$
\frac{1}{2} m \omega^{2}\left(x^{\prime}+\frac{q E}{m \omega^{2}}\right)^{2}-q E\left(x^{\prime}+\frac{q E}{m \omega^{2}}\right) \text {. }
$$

We make rearrangement, finally we get

$$
\frac{1}{2} m \omega^{2} x^{\prime 2}-\frac{1}{2} \frac{(q E)^{2}}{m \omega^{2}} \text {. }
$$

Substituting $\mathrm{Eq}(27)$ into $\mathrm{Eq}(24)$, we easily get

$$
\frac{-\hbar^{2}}{2 m} \frac{d^{2} \psi}{d x^{\prime 2}}+\frac{1}{2} m \omega^{2} x^{\prime 2} \psi=\left(E+\frac{1}{2} \frac{(q E)^{2}}{m \omega^{2}}\right) \psi
$$

$\mathrm{Eq}(28)$ represents the schrodinger equation of simple harmonic oscillator in the variable $x^{\prime}$

The energy eigen values of harmonic oscillator is turns tobe

$$
E_{n}=\left(n+\frac{1}{2}\right) \hbar \omega-\frac{1}{2} \frac{(q E)^{2}}{m \omega^{2}} \text {. }
$$

We observe from Eq. (29) the energy eigen values of harmonic oscillator is consistent with perturbation theory approximation.

\section{Conclusion}

In this paper, we have analyzed the energy eigen value of harmonic oscillator perturbed by electric field. In order 
to carry out the analysis, we have used two independent methods, suchas the perturbation method and time independent schrodinger equation. We have calculated the first order energy correction, second order energy correction, and the exact energy eigen value of harmonic oscillator using time independent schrodinger equation. It has been found that the energy eigen value of harmonic oscillator is the same in two independent methods.

\section{References}

[1] Mojtaba Jafarpour and Davood Afshar,. Calculation of energy eigenvalues for the quantum anharmonic oscillator with a polynomial potential, J.Phys.A : Math.Gen.35: (2002) 87-92.

[2] David J. Griffiths . Introduction to quantum mechanics, $2^{\text {nd }}$ edition .

[3] Nouredine Z. Quantum mechanics, Concepts and Application, $2^{\text {nd }}$ edition.

[4] Safwan A. etal, Journal of Mathematics Research, vol 3, No, 2: may 2011.

[5] Adelaku A. and Abajingin D. Solution of Quantum Anharmonic Oscillator with Quartic Perturbation, Advances in physics Theories and Application, Vol. 24, 2014. 